\title{
Galectin-3 as a Biomarker of Heart Failure in Children with Congenital Heart Disease
}

\author{
Ahmed Abdel Samed Elhewala ${ }^{1}$, Magdey Mohammed Ibrahem ${ }^{2}$, Eslam Samir Abdel Hafez*1 \\ Departments of ${ }^{1}$ Pediatrics and ${ }^{2}$ Medical Biochemistry, Faculty of Medicine, Zagazig University, Sharkia, Egypt \\ *Corresponding author: Eslam Samir Abdel Hafez, E-mail: mosad8rashed@ gmail.com, Mobile: (+20)01024683929
}

\begin{abstract}
Background: Heart failure is defined as a clinical and pathophysiologic syndrome results from ventricular dysfunction, volume, or pressure overload, alone or both. Galectin-3 is a novel biomarker that mediates fibrosis in many organs like the heart that interest in galactin3 as a potential heart failure biomarker that reflects ongoing ventricular remodeling.

Objective: To see if galectin-3 acts as a biomarker of heart failure in congenital heart disease and evaluate if there is a correlation between galectin-3 and severity of HF detected by Ross classification and echocardiogram.

Patients and Methods: The present study included 38 cases with HF due to CHD admitted to PICU and pediatric department at Zagazig University Hospital from July 2018 to July 2019. Patients were selected to exclude heart failure other than congenital heart disease.

Results: Galectin-3 level was significantly higher in children with CHD than age and sex-matched healthy children. There were statistically significant positive correlations between Galectin 3 and ROSS so higher Galectin-3 levels were associated with measures of HF severity. The level was significantly elevated in children with HF in comparison to those without HF, with a significant correlation with the disease severity. Galectin 3 had sensitivity $80 \%$, specificity $100 \%$, PPV $100 \%$ and PPN $71.4 \%$.

Conclusion: Galectin-3 has short term prognosis and can be used as a tool to help in HF diagnosis in children as a marker of disease severity (positive correlation between Ross classification and galectin-3 serum level was proved) and staging patients with acute HF due to congenital heart diseases.
\end{abstract}

Keywords: Heart Failure, Congenital Heart Disease, Galectin-3.

\section{INTRODUCTION}

Heart failure is clinical and pathophysiologic syndrome results from ventricular dysfunction, volume, or pressure overload, alone or both. In children, it is characterized by signs and symptoms, as poor growth, feeding difficulties, respiratory distress, exercise intolerance, and fatigue and is associated with circulatory, neurohormonal, and molecular abnormalities ${ }^{(\mathbf{1})}$.

There are two primary causes of heart failure in children which are over circulation failure occurs when blood mix inside the heart due to a congenital heart defect and pump failure occurs due to damaged heart muscle and can't contracts normally ${ }^{(2)}$.

Diagnosis, risk assessment, and treatment of heart failure are difficult and so need additional tools to improve clinical signs ${ }^{(3)}$.

The classification (ROSS classification) can predict risk from the disease so that management can be tailored to HF class. Children classified as "no risk" not need treatment. "Mild risk" needs close observation, early intervention while "Moderate risk" needs more intensive treatment, and "severe risk" requires maximal therapy and may transplantation referral ${ }^{(4)}$.

Galectin-3 is a novel biomarker that mediates fibrosis in a variety of organs including the heart that had led to the interest of galactin 3 as a potential heart failure biomarker that could reflect ongoing ventricular remodeling ${ }^{(5)}$.

This work aimed to see if galectin-3 acts as a biomarker of heart failure due to congenital heart

disease in children and to evaluate if there is any correlation between galectin-3 and severity of HF as detected by Ross classification and echocardiogram.

\section{SUBJECTS AND METHODS}

1. Type of the study: Case-control.

2. Study setting: The study was conducted in the Pediatric Cardiology Unit, Pediatric Department, Zagazig University Hospitals, Sharkia, Egypt

3. Study period: From July 2018 to July 2019.

4. Sample Size: Total Sample Size included 60 infants, 20 of them were control group, 20 had congenital heart disease without heart failure and the last 20 had congenital heart disease with heart failure.

5. Sample collection and grouping: The target sample comprised 60 randomly chosen infants of age ranged from (6 months - 36 months) selected from those admitted to the Pediatric Cardiology Unit and well diagnosed as having acute heart failure due to congenital heart diseases. 
Inclusion criteria: Age group of infancy $(6 \mathrm{~m}-36 \mathrm{~m})$. Both sex with written consent from the parents. Patients with CHD were diagnosed by Echocardiography.

Exclusion criteria: Infants with HF due to causes rather than congenital heart disease such as cardiomyopathy. Infants with age above 3 years. Other conditions with a high level of galectin 3 such as (cancer and chronic renal failure).

\section{Administrative and ethical design:}

Ethical approval was obtained from the ethical committee review board of the Faculty of Medicine, Zagazig University. The researcher visited the study location, met with the doctors, explained to them the aim and the purpose of the study and its procedures, and asked for their cooperation. Informed consent for participation in the study was taken from the parents or guardians of the participants after a full explanation of the aim of the study. They were informed that participation in the study was voluntary. The parents were allowed to refuse participation. They also assured that any information collected would be confidential and used for the research purpose only.

After approving the protocol by the Hospital Ethics Committee and taking consent from the parents, all patients included in this study were subjected to:

1. Complete history taking through a standardized clinical cardiology sheet with special emphasis on:

- Name, age, sex, birth weight, length, and head circumference.

- Symptoms of heart failure (feedings difficulties, cough, dyspnea, recurrent chest infection, fever, edema, chest wheeze.)

- According to the history taken the patients were classified according to the symptom-based on Modified Ross classification of heart failure ${ }^{(3)}$.

2. General and local examination:

- Vital signs: including
- Heart rate and Respiratory rate assessment: were calculated over one minute.

- Blood pressure, O2sat: were measured and detected

3.

- Local examination of the heart by

4. Investigations:

A. Transthoracic echocardiography (TTE);

B. Chest X-ray (CXR): A chest X-ray was performed for all to detect cardiomegaly, pneumonia by the anteroposterior view.

C. Galectin-3 level: Serum Galectin-3 levels were determined using enzyme-linked immune- sorbent assay (ELISA) kit (Inova, Bioneovan Co., Ltd).

\section{Statistical analysis:}

Recorded data were analyzed using the statistical package for social sciences, version 20.0 (SPSS Inc., Chicago, Illinois, USA). Quantitative data were expressed as mean \pm standard deviation (SD). Qualitative data were expressed as frequency and percentage.

\section{The following tests were done:}

- Independent-samples t-test of significance was used when comparing two means.

- Chi-square $\left(\mathrm{x}^{2}\right)$ test of significance was used to compare proportions between two qualitative parameters.

- The confidence interval was set to $95 \%$ and the margin of error accepted was set to $5 \%$. The p-value was considered significant as the following:

- Probability (P-value)

- P-value $<0.05$ was considered significant.

- P-value $<0.001$ was considered highly significant.

- P-value >0.05 was considered insignificant.

\section{RESULTS}

There was no statistically significant difference between cases and controls regarding age (months) and Sex (Table 1)

Table (1): Comparison between cases and controls regarding demographic data

\begin{tabular}{|c|c|c|c|c|c|c|}
\hline & & & $\begin{array}{c}\text { Cases } \\
(\text { No. }=40) \\
\end{array}$ & $\begin{array}{l}\text { Controls } \\
\text { No. }=20)( \\
\end{array}$ & t.test & P-value \\
\hline \begin{tabular}{|l|} 
Age(months) \\
\end{tabular} & Mean & & $15.48 \pm 10.072$ & $17.05 \pm 9.929$ & 0.574 & 0.568 \\
\hline \multirow{4}{*}{ Sex } & \multirow{2}{*}{ Female } & No. & 15 & 13 & \multirow{4}{*}{$\begin{array}{c}\mathbf{X}^{\mathbf{2}} \\
4.051\end{array}$} & \multirow{4}{*}{0.064} \\
\hline & & $\%$ & $37.5 \%$ & $65.0 \%$ & & \\
\hline & \multirow{2}{*}{ Male } & No. & 25 & 7 & & \\
\hline & & $\%$ & $62.5 \%$ & $35.0 \%$ & & \\
\hline
\end{tabular}

There was no statistically significant difference between cases and controls regarding LVESD and Fs(\%). There was a statistically significant increase in pulmonary pressure and PW among cases than controls as well as a statistically significant decrease in LVEDD and EF among cases than controls (Table 2). 
Table (2): Comparison between cases and controls regarding ECHO

\begin{tabular}{|c|c|c|c|c|c|}
\hline & & $\begin{array}{c}\text { Cases } \\
(\mathrm{No}=40)\end{array}$ & $\begin{array}{l}\text { Controls } \\
(\mathrm{No}=20)\end{array}$ & t. test & P-value \\
\hline LVEDD & Mean \pm SD & $22.50 \pm 11.68$ & $28.10 \pm 1.44$ & $-2.126-$ & .038 \\
\hline LVESD & Mean \pm SD & $19.80 \pm 8.96$ & $17.95 \pm 1.50$ & .912 & .365 \\
\hline EF & Mean \pm SD & $64.22 \pm 13.26$ & $70.40 \pm 3.87$ & $-2.031-$ & .047 \\
\hline pul pressure & Mean \pm SD & $39.60 \pm 9.12$ & $22.60 \pm 2.16$ & 8.192 & .000 \\
\hline PW & Mean \pm SD & $13.35 \pm 8.07$ & $4.04 \pm 0.51$ & 5.134 & .000 \\
\hline $\operatorname{Fs}(\%)$ & Mean \pm SD & $37.17 \pm 7.66$ & $35.40 \pm 2.54$ & 1.005 & .319 \\
\hline
\end{tabular}

There was a statistically significant increase in Galectin 3 (ng/dl) among cases than controls (Table 3).

Table (3): Comparison between cases and controls regarding Galectin 3

\begin{tabular}{|l|l|c|c|l|l||}
\hline \multicolumn{2}{|c|}{} & $\begin{array}{c}\text { Cases } \\
(\text { No.= 40) }\end{array}$ & $\begin{array}{c}\text { Controls } \\
(\text { No.= 20) }\end{array}$ & t.test & P-value \\
\hline Galectin 3(ng/d) & Mean \pm SD & $18.62 \pm 13.056$ & $4.67 \pm 1.97$ & 4.734 & .000 \\
\hline
\end{tabular}

This table shows regarding ROSS 1 (15\%), 2 (37.5\%), 3 (45\%) and 4 (2.5\%) (Table 4).

Table (4): ROSS classification among cases.

\begin{tabular}{||c|c|c|c|}
\hline \multicolumn{2}{|c|}{} & No. & \% \\
\hline \multirow{3}{*}{ ROSS } & $\mathbf{1}$ & 6 & 15.0 \\
\cline { 2 - 4 } & $\mathbf{2}$ & 15 & 37.5 \\
\cline { 2 - 4 } & $\mathbf{3}$ & 18 & 45.0 \\
\cline { 2 - 4 } & $\mathbf{4}$ & 1 & 2.5 \\
\hline
\end{tabular}

There was a statistically significant decrease in Galectin 3 among the cases group without heart failure than the cases group with heart failure (Table 5).

Table (5): Comparison between cases group without heart failure and cases group with heart failure regarding Galectin 3(ng/dl).

\begin{tabular}{||l|c|c|c|c|c|}
\hline \multicolumn{2}{|c|}{} & $\begin{array}{c}\text { ases group without } \\
\text { heart failure } \\
(\mathbf{N o = 2 0})\end{array}$ & $\begin{array}{c}\text { Cases group with } \\
\text { heart failure } \\
(\mathbf{N o = 2 0})\end{array}$ & t.test & P-value \\
\hline glectin 3(ng/dl) & Mean \pm SD & $11.31 \pm 5.59$ & $25.93 \pm 14.35$ & $-4.244-$ & .000 \\
\hline
\end{tabular}

This table shows the value of Galectin-3 with sensitivity $80 \%$, specificity $100 \%$, PPV $100 \%$, and PPN $71.4 \%$ (Table 6 and Figure 1).

Table (6): Validity and predictivity of Galectin-3 in the diagnosis of cases.

\begin{tabular}{|c|c|c|c|c|c|c|}
\hline Variable & Sense \% & Spec\% & PPV\% & NPV\% & AUC & P-value \\
\hline Galectin 3 $\geq \mathbf{1 0}$ & $80 \%$ & $100 \%$ & $100 \%$ & $71.4 \%$ & 0.99 & $<0.001(\mathrm{HS})$ \\
\hline
\end{tabular}




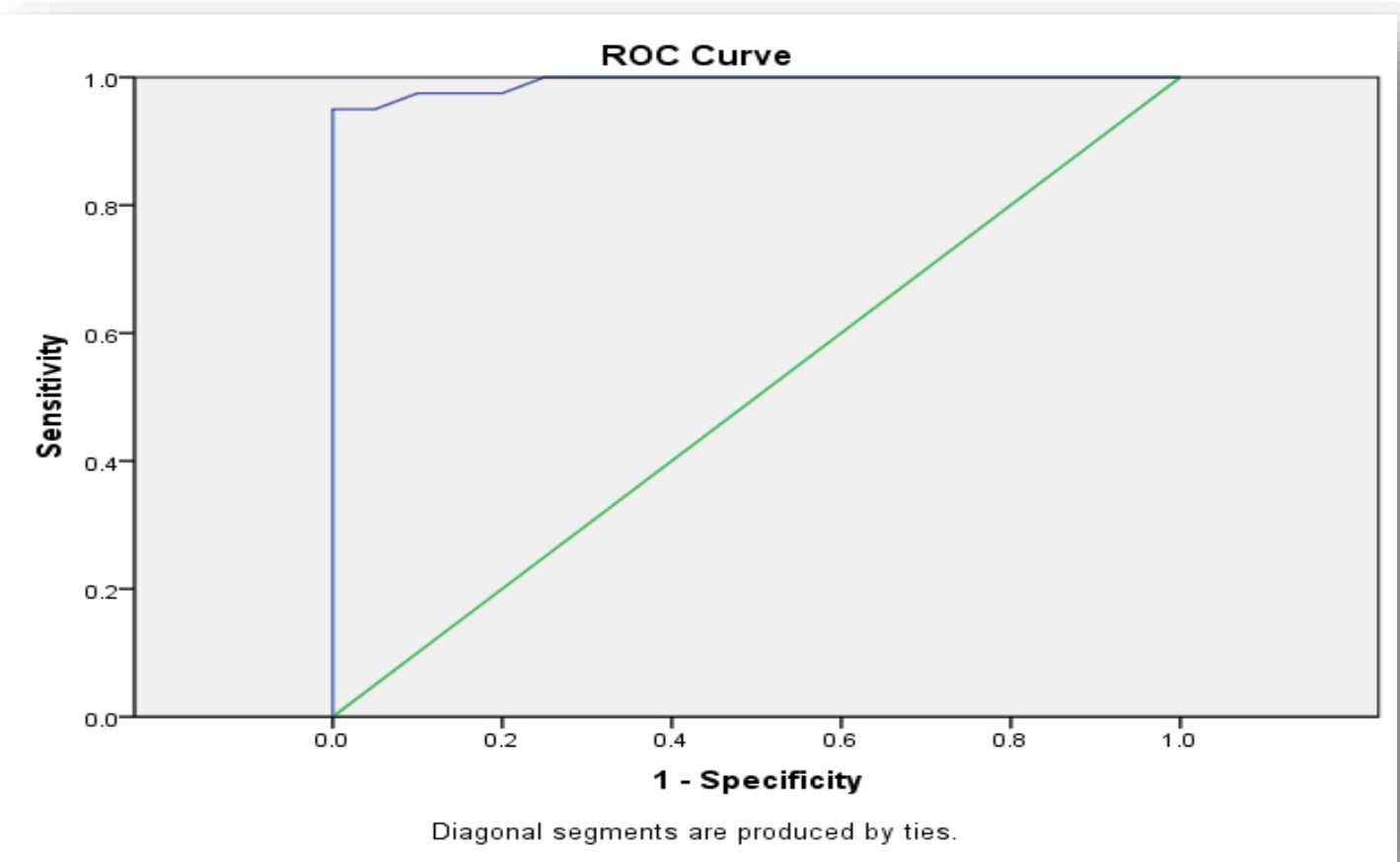

Figure (1): ROC curve of Galectin-3 in the diagnosis of cases

\section{DISCUSSION}

This study showed that according to Echocardiographic data, there was a statistically significant increase in pul pressure and PW among cases than controls. There was a statistically significant decrease in LVEDD and EF among cases than controls.

This agrees with Mohammed $\boldsymbol{e t}$ al. ${ }^{(5)}$ who aimed to investigate the role of Galectin-3 as a marker in children with CHD either with or without HF. Sixty congenital heart disease (CHD) children were chosen randomly. Thirty of them had heart failure (Ross II, III) and the other 30 patients without HF. In addition to 30 age and sex-matched healthy children were chosen as a control group. It was found that there was a statistically significant increase in pul pressure and PW among cases than controls. There was a statistically significant decrease in LVEDD and EF among cases than controls.

This study showed that the Galectin-3 level was significantly higher in children with CHD than age and sex-matched healthy children $(\mathrm{P}<0.001)$. Similar findings were reported in patients with $\mathrm{HF}^{\left({ }^{(6)}\right.}$.

Our study reported that patients with complex cardiac lesions had a statistically significant increase in Galectin-3 serum level (mean Gal-3 level was $16 \mathrm{ng} \backslash \mathrm{ml}$ in patients with complex lesions while it was $9 \mathrm{ng} \backslash \mathrm{ml}$ in patients with a simple lesion, P-value $=0.04$ which was significant).

The results of our study go in agreement with the results of Gopal $\boldsymbol{e t}$ al. ${ }^{(7)}$ who explained that by the fact that galectin-3 concentration has been correlated with markers of the extracellular matrix. Turnover implying a relationship between macrophages phages activation and collagen turnover because galectin-3 is associated with interstitial fibrosis, it seemed likely that galectin-3 might also increase transiently with an episode of decompensation.

Expression of Galectin-3 appears to occur before evident HF and thus may be more useful for the prediction and prevention of disease sequel ${ }^{(\mathbf{8})}$.

Galectin-3 plays an important regulatory role in cardiac fibrosis and remodeling, which are key contributing mechanisms to the development and progression of $\mathrm{HF}^{\left({ }^{(9)}\right.}$.

In rat models, Galectin-3 expression was found to be up-regulated in myocardial biopsies of rats with cardiac hypertrophy that subsequently progressed to $\mathrm{HF}$, and administration of galectin-3 leads to progressive fibrosis and LV systolic dysfunction ${ }^{(10)}$.

These findings were confirmed in human myocardial biopsies, with higher levels of galectin-3 in those with poorer LVEFs ${ }^{(11)}$.

This study showed that there were statistically significant positive correlations between Galectin 3 and ROSS so higher Galectin-3 levels were associated with measures of HF severity.

In adult studies, Galectin-3 levels were higher in HF patients versus controls, and higher galectin-3 levels were associated with measures of HF 
severity, including higher NYHA classification, worse treatment outcome ${ }^{(12)}$, longer hospitalization, and greater mortality ${ }^{(13)}$. This relationship is presented in both HF with reduced or preserved $\mathrm{EF} \%$ (2) and even in acute HF ${ }^{(14)}$. Additionally, elevated Galectin-3 could predict cardiovascular adverse events and incident HF in healthy populations ${ }^{(15)}$.

In our study, the Galectin-3 level was significantly elevated in children with $\mathrm{HF}$ in comparison to those without $\mathrm{HF}$, with a significant correlation with the disease severity.

There is limited data about serum Galectin3 level in children, in clinical studies, serum where it ranged between $0-1.8 \mathrm{ng} / \mathrm{ml}$ among neonates, while in older children its level ranged between 0-2.14 ng/ml). In the present work, serum Galectin-3 level was ranged between 2-33.9 $\mathrm{ng} / \mathrm{dl}$.

Regarding serum Galectin-3 level in our study, its mean values were $14.2 \mathrm{ng} / \mathrm{ml}$, which was higher than values in a pediatric study on heart failure by Kotby et al. ${ }^{(17)}$ who reported a mean value of serum Galectin-3 levels among the patient's groups was 9.4. This difference can be explained by the different patient populations and different assays used between different studies.

Gopal et al. ${ }^{(7)}$ had demonstrated that Galectin-3 levels were similarly elevated in all patients with HF, regardless of whether it was acute or chronic or it was systolic or diastolic.

In our study, there were statistically significant positive correlations between Galectin 3 and (pul pressure, SBP, DBP, and ROSS). While there were statistically significant negative correlations between Galectin-3 and ( $\mathrm{Hb}, \mathrm{O} 2$ sat and weight $(\mathrm{kg})$. There were no statistically significant correlations between galectin 3 and other numerical data. In adults, elevated Galectin-3 was associated with echocardiographic parameters representative of impaired cardiac function and unfavorable remodeling (18).

Additionally, the Galectin-3 expression is modulated by genetic variances ${ }^{(2)}$. These data could explain the previous conflicting results. Further studies at a larger scale of patients are required to reach an agreed prognostic cut-off value for galectin3 in children with HF.

This study showed that, the value of Galectin-3 with sensitivity $80 \%$, specificity $100 \%$, PPV $100 \%$, and PPN 71.4\%.

Chen et al. ${ }^{(19)}$ demonstrated that Galectin-3 level had $62.9 \%$ sensitivity and $90 \%$ specificity to predict congestive heart failure (CHF).

This study showed that, the percentage of Ross HF classification $1=(15 \%)$, the percentage of Ross HF classification $2=(37 \%)$, the percentage of Ross HF classification $3=(45 \%)$ and the percentage of Ross HF classification $4=(2.5 \%)$
The Ross HF classification was developed to assess severity in infants and has subsequently been modified to apply to all pediatric ages. The modified Ross classification for children provides a numeric score ${ }^{(20)}$.

Our study showed that there was no statistically significant association between galectin-3 serum level and the presence of cyanosis in studied patients. which agreed with Kotby et al. ${ }^{(17)}$ who reported that there was no statically significant difference in serum galectin-3 level among patients with different HF etiologies.

In our study there was no significant correlation was found between serum galectin-3 levels and patients' systolic and diastolic blood pressure ( $\mathrm{P}=0.06,0.2$ respectively) which agreed with Kotby $\boldsymbol{e t}$ al. ${ }^{(17)}$.

A statistically positive correlation in our study was found between serum Galectin-3 level and Ross HF class classification $(\mathrm{P}$-value $=0.04)$ which agreed with Kotby et al. ${ }^{(17)}$ (P-value in his study was less than 0.001).

Accumulating data supporting the idea that apoptosis is involved in the transition from cardiac compensation to decompensated congestive heart failure and galectin-3 is involved in apoptosis. Thus heart failure decompensation may be promoted as a Galectin-3 level increase ${ }^{(5)}$.

Also, Iqbal et al. ${ }^{\left({ }^{(8)}\right.}$ showed that Galectin-3 concentrations have been correlated with markers of extracellular matrix turnover implying a relationship between macrophage activation and collagen turnover.

Gopal et al. ${ }^{(7)}$ reported that Galectin-3 is associated with interstitial fibrosis, it seemed likely that plasma Galectin-3 might also increase transiently with an episode of decompensation

In the present study, a ROC curve analysis was performed to evaluate the predictive value of serum Galectin-3 for short term diagnosis in acute heart failure patients.

However, Kotby et al. ${ }^{(17)}$ ROC curve was used to define the best cutoff value of galectin-3 for differentiating patients and controls, which was more than 3 with sensitivity of $100 \%$, specificity of $79.8 \%$ and diagnostic accuracy of $100 \%$.

While, Mohammed et al. ${ }^{(5)}$ ROC curve analysis was carried out to identify the optimal cut off point of Galectin-3 concentration for HF diagnosis which was higher than 1.035 with a sensitivity of $83.88 \%$, specificity of $63.33 \%$ and $80 \%$.

\section{CONCLUSION}

Galectin-3 has a role in short term prognosis and can be used as a tool to help in HF diagnosis in children as a marker of disease severity (positive correlation between Ross classification and galectin-3 
serum level was proved ) and staging in patients with acute HF due to congenital heart diseases.

\section{REFERENCES}

1. Kirk R, Dipchand AI, Rosenthal DN et al. (2014): The International Society for Heart and Lung Transplantation Guidelines for the management of pediatric heart failure: Executive summary (Corrected). The Journal of heart and lung transplantation: the official publication of the International Society for Heart Transplantation, 33(9): 888.

2. De Boer RA, Lok DJ, Jaarsma T et al. (2011): Predictive value of plasma Galectin-3 levels in heart failure with reduced and preserved ejection fraction. Ann Med., 43: 608.

3. Ross RD (2012): The Ross classification for heart failure in children after 25 years: a review and an age-stratified revision. Pediatr Cardiol., 33: 1295-1300.

4. Wu AH, Wians F, Jaffe A et al. (2013). Biological variation of Galectin-3 and soluble ST2 for chronic health failure: implication on the interpretation of test results. Am Heart J., 165(6):995-999.

5. Mohammed LA, Gafar HS, Hussien NR et al. (2014): Galectin-3 as an early detector of heart failure in children with congenital acyanotic heart disease. Clinical Medicine and Diagnostics, 4 (5): 90-98.

6. Milting H, Ellinghaus P, Seewald M et al. (2008): Plasma biomarkers of myocardial fibrosis and remodeling in terminal heart failure patients supported by mechanical circulatory support devices. J Heart Lung Transplant, 27: 589-596.

7. Gopal DM, Kommineni M, Ayalon $\mathrm{N}$ et al. (2012): Relationship of plasma Galectin-3 to renal function in patients with heart failure: effects of clinical status, the pathophysiology of heart failure, and presence or absence of heart failure. J Am Heart Assoc., 1: 000760.

8. Iqbal $\mathrm{N}$, Wentworth $\mathrm{B}$, Choudhary $\mathrm{R}$ et al. (2012): Cardiac biomarkers: new tools for heart failure management. Cardiovasc Diagn Ther., 2(2): 147-164.
9. Radziwolek L, Radunski UK, Koopmann K et al. (2014): Myocardial injury and fibrogenesis: extracellular volume expansion is associated with elevated Galectin-3 levels in patients with myocarditis. Journal of Cardiovascular Magnetic Resonance, 16(1): 290-295.

10. Sharma UC, Pokharel S, van Brakel TJ et al. (2004): Galectin-3 marks activated macrophages in failure-prone hypertrophied hearts and contribute to cardiac dysfunction. Circulation, 110:3121-3128.

11. De Filippi D, Felker $M$ (2010): Galectin-3 in Heart Failure-Linking Fibrosis, Remodeling, and Progression. USA Cardiology, 7(1): 67-70.

12. Khaity M, Al-Quobaili F (2013): Utility of Plasma Galectin-3, a Novel Marker of Fibrosis, for Evaluation of Syrian Patients with Chronic Heart Failure. Int J Pharm Sci Rev Res., 21(2): 287-292.

13. van der Bom T, Winter MM, Bouma BJ et al. (2012): The effect of valsartan on the systemic right ventricular function: a double-blind randomized placebo-controlled pilot trial. Circulation, 127(3):322-30.

14. Van Kimmenade RR, Januzzi JL, Ellinor PT et al. (2006): Utility of amino-terminal pro-brain natriuretic peptide, galectin-3, and apelin for the evaluation of patients with acute heart failure. J Am Coll Cardiol., 48:1217-1224.

15. Djoussé L, Petrone AB, Gaziano JM (2015): Consumption of fried foods and risk of heart failure in the physicians' health study. Journal of the American Heart Association, 4(4): e001740.

16. Demmert M, Faust K, Bohlmann MK et al. (2014): Glacetin in cord blood of term and preterm infants. Clinical and Experimental Immunology, 167:246-251.

17. Kotby AA, Youssef OI, Elmaraghy MO et al. (2017): Galectin-3 in children with chronic heart failure with normal and reduced Ejection Fraction: Relationship to Disease severity. Pediatr Cardiol., 38(1):95-102.

18. Shah RV, Januzzi JL (2014): Soluble ST2 and Galectin-3 in Heart Failure. Clin Lab Med., 34:87-97.

19. Chen D, Assad-Kottner C, Orrego C et al. (2008): Cytokines and acute heart failure. Crit Care Med., 36: 9-16.

20. Jayaprasad $\mathbf{N}$ (2016): Heart failure in children. Heart Views, 17(3): 92-99. 